\title{
Influence of pattern variance and overt responding on subjective categorization in a two-schemata SCF task'
}

\author{
MARGARET A. HASTINGS, D. F. \\ DANSEREAU, and T. R. DIXON, Texas \\ Christian University, Fort Worth, Tex. \\ 76129
}

Forty patterns representing two schemato each at four redundancy levels were generated by a VARGUS 9 procedure and presented to $S s$ for categorization. Ss were able to provide scaled judgments of pattern variability both within and between schemata. Performance on a subsequent constrained sorting task was not a function of overt vs covert responding in the scaling task.

Developed as an extension of the schema plus correction encoding hypothesis discussed by Woodworth (1938), Oldfield (1954), and Attneave (1957), schema theory provides a conceptual and methodological framework for the construction of theories of pattern perception (Evans, 1967a). Within this theory a schema family is considered to be a cluster of points in multidimensional space. The prototype of the family consists of the communalities between members of the cluster (i.e., the centroid). In order to provide a methodological basis for experimentation, a number of computer-generation procedures have been developed to produce probabilistically determined schema families (Evans \& Mueller, 1966; Evans, 1967c). For this class of stimuli, the degree to which a stimulus population adheres to the prototype or schema rule (i.e., tightness of the cluster) is under experimental control.

It is assumed that naturally occurring categories defined in terms of clusters of covarying characteristics exist in the real world (work in automatic pattern recognition seems to support this, e.g., Cooper, 1968). To cope with such a world, organisms are postulated to have the capacity to recognize objects within a heterogeneous environment according to their schematic classifications. It is further hypothesized that people can detect these schema-defined categories without any information except that which the environment itself provides (Evans, 1976a). Unlike traditional concept formation research, schematic classification does not require knowledge of results.

If the schema plus correction encoding hypothesis is tenable, humans must be able to encode some type of pattern-specific correction as well as the prototype itself. One requirement of this hypothesis is that Ss be able to discriminate stimuli on the basis of their distance from the schema. Although the level of distortion of stimuli from a prototype has been shown to affect schematic categorization, there has been no analysis of the S's ability to order stimuli within schema families according to their variability. Moreover, investigations of the relationship between pattern distortion and subjective categorization have generally relied upon dichotomous (same-different) response measures. The present study was designed to determine whether Ss could respond to both schematic categories and level of distortion within schema (i.e., make a more sensitive classification than a dichotomous response would allow). It was of interest to determine if the subjective basis for categorization would be correlated with PV, a pattern-specific physical distance measure of pattern distortion from a prototype (see Evans \& Mueller, 1966).

The experiment consisted of two parts, Phase 1, a classification and scaling task, and Phase 2, a constrained sorting task. During Phase 1, Ss were required to classify computer-generated stimuli into both appropriate schema families and appropriate distortion levels within schema. Half of the Ss classified patterns by making an overt response (overt responders), and half of the $\mathrm{Ss}$ made no overt classification (covert responders).

The influence of overt vs covert responding upon schematic performance was investigated in Phase 2. In this phase, Ss were asked to sort the same stimulus patterns into two classes. By requiring all $\mathrm{Ss}$ to participate in the constrained sorting task during Phase 2 of the experiment, the effect of the Phase 1 response requirement (overt or covert) could be evaluated.

\section{SUBJECTS}

The Ss were 28 students enrolled in two undergraduate psychology courses at Texas Christian University. Ss were randomly assigned to the "overt" and "covert" treatment conditions as they came to the experiment. The two treatment conditions were run simultaneously.

\section{STIMULI}

The patterns used as stimuli were produced by a VARGUS 9 computer system (Evans \& Mueller, 1966). The VARGUS 9 system generates randomly sampled stimuli from a population of patterns constituting a schema family. As discussed in detail by Evans (1967b), the statistical characteristics of the schema family (tightness of the multidimensional cluster about the prototype) are a function of the population parameter, constraint redundancy (Rc). Lowering Rc reduces the degree to which the stimuli resemble the prototype and concommitantly raises the difficulty level of schematic classification (Evans, 1967b). The two prototypes were constructed to achieve a high degree of discriminability between the two schema families.

Employing the VARGUS 9 program, five stimuli at each of three levels of redundancy $(70 \%, 50 \%$, and $40 \%)$ were generated for each of two schemata. An additional five $100 \%$ redundant stimuli used for each schema family were the prototypes themselves. All patterns were presented on slides in a black polygon format (see Fig. 1). These 40 stimuli, 5 at each of the four redundancy levels for each of the two schemata, constituted the stimulus materials. For purposes of stimulus presentation, three random orders of the $\mathbf{4 0}$ stimuli were constructed with the constraint that each block of 8 stimuli contain all eight combinations of schema by redundancy level. Each stimulus was presented for $15 \mathrm{sec}$. Eight additional stimuli representing the two schemata at each of the four redundancy levels were prepared as examples of correct categorization. These stimulus examples were grouped by schema on a pottern example sheet such that they

\section{PATTERN EXAMPLES}

GROUP A
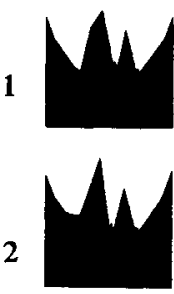

3
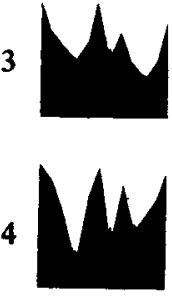

GROUP B
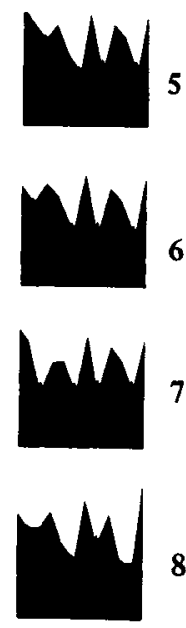

Fig. 1. From top to bottom, Schema $A$ and Schema $B$ at four levels of redundancy $-100 \%, 70 \%, 50 \%$, and $40 \%$. 


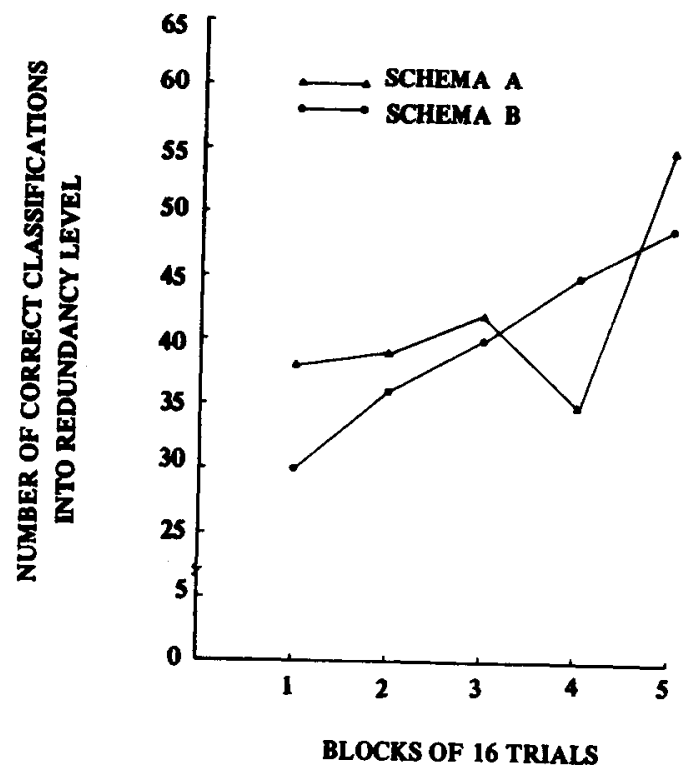

reflected increasing amounts of deviation from the prototype (see Fig. 1).

TASK AND PROCEDURE

Both the "overt" and the "covert" groups simultaneously observed the stimuli in Phase 1, the initial scaling task. All conditions were identical for both groups except that the latter was not required to make an overt judgment. The instructions provided each $S$ with a common example of pattern discrimination, the classification of handwriting samples. The task was presented as an example of unsupervised pattern classification; no knowledge of results was provided. In addition to instructions, each $S$ received a copy of the example sheet. This sheet provided the eight possible pattern-classification al ternatives.

The task was presented as a two-part assignment. During the $15-\mathrm{sec}$ exposure time, each stimulus was to be categorized first into one of the two major groupings, $A$ or B (on the pattern example sheet), and then into one of the four subgroupings within the class. The basis for categorization was a physical similarity criterion. No mention of redundancy or variability about a prototype was made. A single number, from 1 to 8 , indicated both classification decisions for each pattern (1.4 represented increasing deviations from Pattern $A$, and 5-8 represented increasing deviations from Pattern B). The two prototypes, A and B, appeared at the top of the pattern example sheet. Under each were stimuli drawn from the three levels of redundancy from high to low (again see Fig. 1). During Phase 1, 80 patterns were presented automatically at $15 \mathrm{sec} / \mathrm{stimulus}$ via Carousel projector. The same 40 stimuli were presented in two random orders to each $S$ in order to provide an estimate of classification reliability.
During Phase 2 of the experiment, all Ss were required to respond overtly. Ss were told that the patterns which they had previously seen would be presented again. This time, however, they were to sort them into two groups, $\mathrm{A}$ and $\mathrm{B}$, on any basis they chose. Pattern example sheets were not provided. The 40 stimuli were then presented in a third random order. Exposure time was again $15 \mathrm{sec}$ and automatically controlled.

The dependent variables used as measures of performance in the two tasks were: (1) Phase 1-number of stimuli classified into both the correct major grouping (Schema Family A or B) and the correct variability subgrouping within schema ( redundancy level), and (2) Phase 2-number of stimuli classified into correct schema family (correct A or B grouping). Additionally, Phase 1 responses were transformed from the original two-dimensional scale (representing schema family and redundancy level as separate dimensions) to a unidimensional scale in which Ss' responses represented judgments of distance between the stimulus pattern and the pattern prototype. This transformation allowed an assessment of the correlation between PV (pattern deviation from prototype) and subjective categorization on an unidimensional distance scale.

\section{RESULTS}

In Phase 1 , a two-way analysis of variance (with factors schema type and blocks of trials) performed on the number of stimuli classified into both the appropriate schema family and the correct redundancy level yielded a significant trials effect $(F=4.617$, $\mathrm{df}=4,52, \mathrm{p}<.005)$. For overt responders,
Fig. 2. Classification of patterns into correct redundancy level within schema. Maximum of 112 correct classifications per block of 16 trials.

correct classification of stimuli by redundancy level increased over trials (see Fig. 2). Neither the schema effect nor the Schema by Trials interaction was significant.

Two considerations, however, made this analysis less than satisfactory. First, redundancy levels are not mutually exclusive classes. This makes the assignment of particular patterns to a single redundancy level impossible. Second, the two schemata used in this research were not identical in difficulty, i.e., the obtained mean PV for patterns within a particular level was not consistent across schemata. Given this unrepresentative pattern sample, it was more appropriate to consider each pattern as representing a particular amount of deviation from its prototype rather than as representing a redundancy class. In view of these considerations, $\mathrm{Ss}^{\prime}$ responses were transformed onto an 8-point unidimensional scale reflecting subjective judgments of physical distance or deviation between the stimulus pattern and the schema prototype. The larger the number assigned to the stimulus on this scale, the greater the judged deviation between the presented pattern and the prototype. The numbers 1-4 represented a correct schematic classification, while the numbers 5.8 represented an incorrect schematic classification. Thus, a judgment of 8 on the unidimensional distance scale indicated that a Schema $A$ stimulus had been judged to be a Schema B prototype. Employing this transformation, a relationship between subjective categorization of stimuli along a physical distance scale and PV could be ascertained. A Pearson product-moment correlation between the transformed classification judgments and PV yielded an $r$ of .75 for the first presentation order of the $\mathbf{4 0}$ stimuli and an $\mathrm{r}$ of .73 for the second order. Reliability of stimulus classification for the two orders was 94.

A $t$ test comparing mean correct schematic classifications for overt and covert responders during Phase 2 of the experiment was nonsignificant. From a total of 40 possible correct schematic classifications, the mean and variance of the overt responders was 33.9 and 3.9 , while that of the covert responders was 33.2 and 3.5. This similarity suggests the tentative conclusion that under the conditions of the present experiment, the requirement of overt or covert responding does not contribute to differences in schematic classification performance. 


\section{DISCUSSION}

The present study demonstrates Ss' capacity to respond both to schematic categories and to levels of distortion within schemata. This finding supports previous research indicating that humans can employ probabilistic regularities as a basis for subjective categorization (Evans \& Edmonds, 1966; Evans \& Amoult, 1967; Brown, Walker, \& Evans, 1968). In addition, Ss appear sensitive to subgroupings defined in terms of pattern variability within schema clusters. Although the task was not designed to facilitate subjective scaling along a physical distance dimension, the correlation between subjective categorization and physical distance from prototype accounted for $55 \%$ of the variance. Ss' sensitivity to small differences in variability from a prototype as measured by PV appears to be acute. This conclusion is corroborated by Ss' performance in a free-sorting task employing VARGUS 7 stimuli. ${ }^{2}$ It is noteworthy, however, that both the pattern example sheet and the instructions provided information about (1) the number of schemata present and (2) the hierarchical arrangement of patterns within schema. Although subjective classification of stimuli into the appropriate schema family and the correct redundancy level improved significantly over trials, the reliability of stimulus assignment on the 8-point scale was extremely high (.94) over the two halves of the task. These data suggest, therefore, that while Ss improved their ability to categorize stimuli according to variability levels, patterns retained their relative ordinal positions in terms of subjective categorization. It can be concluded, therefore, that improvement over trials was general in nature rather than specific to a particular level of distortion. A more finely graded response scale, however, would provide a more sensitive measure of possible changes in either sensitivity to variability or classification criterion as a function of schematic concept formation.
Failure to find a difference in the Phase 2 task as a function of the Phase 1 response requirement suggests that under the conditions of the present experiment overt responding neither facilitates nor impedes schematic classification.

\section{REFERENCES}

ATTNEAVE, F. Transfer of experience with a class-schema to identification-learning of patterns and shapes. Journal of Experimental Psychology, 1957, 54, 81-88.

BROWN, B. R., WALKER, D. W., \& EVANS, S. H. Schematic concept formations as a function of constraint redundancy and knowledge of results. Psychonomic Science, 1968, 11, 75-76.

COOPER, P. W. Non-supervised learning in adaptive statistical pattern recognition. Paper presented at IFIP Congress 68, Edinburgh, Scotland, August 1968.

EVANS, S. H. A brief statement of schema theory. Psychonomic Science, 1967a, 8, 87-88.

EVANS, $S$. H. Redundancy as a variable in pattern perception. Psychological Bulletin, 1967b, 67, $104-113$.

EVANS, S. H. VARGUS 7: Computed patterns from Markov processes. Behavioral Science, $1967 \mathrm{c}, 12,323-328$.

EVANS, S. H., \& ARNOULT, M. C. Schematic concept formation: Demonstration in a free sorting task. Psychonomic Science, 1967, 9, 221-222.

EVANS, S. H., \& EDMONDS, E. M. Schema discrimination as a function of training. Psychonomic Science, 1966, 5, 303-304.

EVANS, S. H., \& MUELLER, M. R. VARGUS 9: Computer stimuli for schema research. Psychonomic Science, 1966, 6, 511-512.

OLDFIELD, R. C. Memory mechanisms and the theory of schemata. British Journal of Psychology, 1954, 45, 14-23.

WOODWORTH, R. S. Experimental psychology. New York: Holt, 1938.

\section{NOTES}

1. This research was supported by the Department of Defense, Project THEMIS Contract (DAAD05-68-C-0176), under the Department of the Army to the Institute for the Study of Cognitive Systems through the TCU Research Foundation. Further reproduction is authorized to satisfy needs of the U.S. Government.

2. Bersted, C. T., Brown, B. R., \& Evans, S. H. Free sorting with stimuli clustered in a multidimensional attribute space, unpublished manuscript.
For the normal infants studied here, the more intense stimulus produced a lustier cry, but latency was not affected.

In previous studies (Karelitz \& Fisichelli, 1962; Fisichelli \& Karelitz, 1963) designed to study the diagnostic potential of the cry in normal infants and those with diffuse brain damage, it was found that the normal infant generally responded more quickly and productively to painful stimulation than the brain-damaged infant. The stimulus was a snap of a rubber band on a gun-shaped apparatus against the sole of the foot. The stimulus, a No. 32 rubber band, and procedure are described in detail in the papers already mentioned.

The present study was designed to explore further the responsivity of normal infants to two stimuli differing in the strength of their impact force. While it may seem obvious that a more intense reaction will be obtained from a more painful stimulus, clinical observation reveals that infants differ in the degree to which their reactions are heightened. The differential in responsivity to two stimuli of varying intensity might provide an additional diagnostic parameter. Parmalee (1962), has already suggested that, among other things, "a 'good cry' ... has a duration proportional to the degree of stimulation ...."

Preliminary findings in a test situation in which a "stronger" rubber band was applied after a "weaker" one indicated that reactions to the "stronger" were more severe than to the "weaker." It remained necessary to determine, however, that the more intense reaction was not merely a summative effect but unique to the "stronger" band. Evidence for this is already available since past observations with successive applications of the "weaker" stimulus do not show heightened responses. The present study proposes a more direct test of the issue and suggests an objective measure of the strengths of the so-called "weaker" and "stronger" stimuli.

\section{METHOD}

The stimuli used were rubber bands of different lengths, No. 32 and the shorter No. 30 of the Springline Parr Amber make. The No. 30 has more sting to it. Since neither the manufacturer nor their testing consultants could provide any quantitative specifications on the "strengths" of their products, the authors devised their own measure of the impact force of the bands. A plastic bottle, measuring $72 \mathrm{~mm}$ long, $48 \mathrm{~mm}$ wide, and $85 \mathrm{~mm}$ high, was filled with water so that the entire unit with cap weighed $150 \mathrm{~g}$. This was placed on a stainless steel surface and the tip of the gun-shaped apparatus with band in cock position was placed against it. The band was released and 